\title{
Relative frequency and prognosis of vascular aphasia (follow-up at 3 months) in the Neurology Department of Assiut University Hospital
}

Hamdy Naguib El-Tallawy ${ }^{1}$, Adel Hassanein El Sayed Gad ${ }^{2}$, Anwar Mohamed Ali ${ }^{1}$ and Manar Nasr Abd-El-Hakim ${ }^{1 *}$ (D)

\begin{abstract}
Background: Post-stroke aphasia (PSA) is a disabling complication after stroke leading to impairment of quality of life. It was reported to develop in one third of patients with stroke and in two thirds of patients with right hemiplegia due to stroke.

Objectives: The aim of this study is to determine the relative frequency, clinical types, and prognosis of vascular aphasia in patients with acute cerebrovascular stroke.

Methods: The study was conducted on 1508 patients who were admitted with acute cerebrovascular stroke to the Neurology Department of Assiut University Hospital during the 6-month period. Among them, there were 107 patients presented with aphasia who were subjected to clinical assessment, Kasr El-Eini Arabic Aphasia test (KAAT), computerized tomography, and/or magnetic resonance imaging of the brain. Follow-up study of aphasic patients was done after 1 and 3 months with KAAT.

Results: Relative frequency of vascular aphasia in the Neurology Department of Assiut University Hospital was 7.1\% and more common among male patients (57.9\%), with the most frequent type was global aphasia (66.4\%). Better improvement in follow-up was for aphasia without repetitive disorder $(p=0.000)$ mainly for subcortical aphasia $(p=0.000)$, and better recovery was reported among younger age groups $(p=0.041)$, patients with cerebral hemorrhage $(p=0.019)$, and patients with small-sized lesions ( $p=0.031)$.
\end{abstract}

Conclusion: There were many prognostic factors which can predict rate of aphasia recovery like age of the patient, aphasia type, size, site, and pathology of the lesion.

Keywords: Aphasia, Stroke, Prognosis, Recovery

\section{Introduction}

Aphasia is an impairment of language, affecting the production or comprehension of speech and the ability to read or write. Aphasia is always due to injury to the brain areas involved in language most commonly from vascular disorders [1].

Post-stroke aphasia (PSA) is a common serious consequence that results from injury to an extended network of cortical and subcortical structures perfused by the middle cerebral artery in the left hemisphere [2], leading

\footnotetext{
* Correspondence: mannarnasr@gmail.com

${ }^{1}$ Neurology Department, Faculty of Medicine, Assiut University, Assiut, Egypt Full list of author information is available at the end of the article
}

to increased long-term morbidity and mortality with residual disability $[3,4]$. It also severely affects the ability to communicate and participation in social activities [5].

The incidence of PSA had been reported to be $40-60$ per 100,000 individuals per annum [6, 7]. PSA is more common for older patients [8]. Fifteen percent of individuals under the age of 65 experience aphasia after their first ischemic stroke; this percentage increases to $43 \%$ for individuals 85 years of age and older [9].

The aim of the work is to determine the relative frequency, clinical types, and prognosis of vascular aphasia in patients with acute cerebrovascular stroke in the Neurology Department of Assiut University Hospital. 


\section{Subjects and methods}

The study is a cohort prospective study conducted in Assiut University Hospital. One thousand five hundred eight patients (1508), 779 males and 729 females, were admitted with acute cerebrovascular stroke (within 2 weeks from onset) to the Neurology Department of Assiut University Hospital, Assiut, Egypt, during the 6month period (June 1, 2015 to November 30, 2015).

Among them, there were 107 (7.1\%) patients presented with PSA, 62 (57.9\%) males and 45 (42.1\%) females, with 33 patients who could not complete the follow-up study (30 patients died and 3 patients could not be accessed).

The study included patients presented with disturbance of language, which was acquired in patients with acute cerebrovascular stroke who were admitted within 2 weeks from onset; also consciousness was clear that it could be judged from patients' behavior, attention to environment, and reaction to sensory stimulation; and the study excluded the thematic context of language [10] and patients with history of mental deterioration, psychiatric disorder, or severe hearing and/or visual impairment.

All selected patients were evaluated within 2 weeks after stroke onset and followed up at periodic intervals (1 month and 3 months later).

Each patient was subjected to history case taking, general medical and neurological examination according to the neurological special questionnaire of the Neurology Department of Assiut University Hospital and neurolinguistic tests: Kasr El-Eini Arabic Aphasia test (KAAT), applied by Hassanein as a simple, rapid, standardized, valid, and reliable bedside test for Egyptian patients, literate and illiterate, for evaluating aphasia [11].

The KAAT consists of six parts: repetition, comprehension (including auditory comprehension, written words, token test, and pantomime), spontaneous speech (including communication, articulation and prosody, automatized language, semantic structure, syntactic structure, verbal output, initiation and effort, and phrase length), naming, reading, and writing (including composition, dictation, copying and spelling). With a total score of 90 for educated and 70 for illiterate patients (after removing comprehension of written words, reading, and writing subtests).

The aphasic patients were classified according to Benson and Geshwind [12] into two groups: aphasia with repetitive disorder (including global, Broca's, Wernicke, conduction aphasia, pure ward deafness, and aphemia) and aphasia without repetitive disorder (including striatal, thalamic, mixed transcortical, transcortical motor, transcortical sensory aphasia, and anomia).

Neuroimaging studies also were done in the form of a computerized tomography (CT) and/or magnetic resonance imaging (M R I) of the brain. CT scan was done using Toshiba Multislice 16 computed tomography, Japan.
MRI scan was obtained by using 1.5 Tesla Siemens Era machine 24 channels, Germany. T2, FLAIR, T1, and diffusion weighted images in the sagittal and axial planes were obtained. The size of cerebral infarction was classified according to Brott and colleagues [13] to small-sized lesion (lesion < half lobe), medium-sized (lesion involving between half lobe and one lobe), and large-sized (lesion > one lobe).

Follow-up study was done by clinical assessment of patients and KAAT test after 1 and 3 months.

An informed consent was obtained from all the patients before participating in the study. The Assiut Faculty of Medicine Ethical committee approved this study in May 24,2015 . The confidentiality of patient's information was maintained during all steps of the study.

\section{Statistical analysis}

Data entry and analysis were done using IBM SPSS statistics (Statistical Package for Social Science, version 22, 2013; IBM Corp., Armonk, NY, USA). Qualitative data were presented as numbers and percentages, and quantitative data were presented as means \pm standard deviation (SD). Chi-square and Fisher Exact tests were used to compare between qualitative variables. Mann-Whitney test was used to compare quantitative variables between two groups, and Kruskal Wallis Test for more than two groups. Wilcoxon signed rank test was done to compare quantitative variables between baseline and follow-up. Regarding statistical analysis for rate of aphasia recovery, the study take in consideration the total score percentage instead of the total score of KAAT test to eliminate the variation between educated and illiterate patients. $P$ value was considered statistically significant when $P<0.05$ and highly significant when $P<0.01$.

\section{Results}

The study was conducted on 1508 patients, 779 (51.66\%) males and 729 (48.34\%) females, with acute stroke. Among them, there were 107 (7.1\%) patients presented with aphasia, 62 (57.9\%) males and 45 (42.1\%) females. Their age ranged from 20 to 85 years with a mean 59.38 years \pm 15.43 SD. Hypertension and age $\geq 55$ years were the most frequent risk factors, and most of the patients had more than one risk factor.

The clinical types of aphasia were classified into two groups, aphasia with repetitive disorder (79.4\%) and aphasia without repetitive disorder (20.6\%) with the most frequent types were global, Broca's then subcortical aphasia (Table 1).

There were 21 patients who had cerebral hemorrhage (19.6\%), 86 patients had cerebral infarction (80.4\%) who were classified according to size of infarction to smallsized lesions in 9 patients, medium-sized in 6 patients, and large-sized in 71 patients (Table 2). 
Table 1 Clinical types of aphasia

\begin{tabular}{|c|c|c|c|c|c|c|c|}
\hline \multirow[t]{2}{*}{ Clinical type } & \multicolumn{2}{|c|}{$\begin{array}{l}\text { Total } \\
(N=107)\end{array}$} & \multicolumn{2}{|c|}{$\begin{array}{l}\text { Male } \\
(N=62)\end{array}$} & \multicolumn{2}{|c|}{$\begin{array}{l}\text { Female } \\
(N=45)\end{array}$} & \multirow[t]{2}{*}{$P$ value* } \\
\hline & $\bar{N}$ & $\%$ & $\bar{N}$ & $\%$ & $N$ & $\%$ & \\
\hline Aphasia with repetitive disorder: & 85 & 79.4 & 47 & 75.8 & 38 & 84.4 & \\
\hline Global & 71 & 66.4 & 41 & 66.1 & 30 & 66.7 & 0.954 \\
\hline Broca's & 9 & 8.4 & 3 & 4.8 & 6 & 13.3 & 0.162 \\
\hline Wernicke & 3 & 2.8 & 2 & 3.2 & 1 & 2.2 & 0.756 \\
\hline Conduction & 1 & 0.9 & 0 & 0.0 & 1 & 2.2 & 0.421 \\
\hline Aphemia & 1 & 0.9 & 1 & 1.6 & 0 & 0.0 & 0.392 \\
\hline Aphasia without repetitive disorder: & 22 & 20.6 & 15 & 24.2 & 7 & 15.6 & \\
\hline Striatal & 12 & 11.2 & 8 & 12.9 & 4 & 8.9 & 0.516 \\
\hline Thalamic & 5 & 4.7 & 2 & 3.2 & 3 & 6.7 & 0.648 \\
\hline Mixed transcortical & 2 & 1.9 & 2 & 3.2 & 0 & 0.0 & 0.508 \\
\hline Transcortical motor & 1 & 0.9 & 1 & 1.6 & 0 & 0.0 & 0.392 \\
\hline Transcortical sensory & 1 & 0.9 & 1 & 1.6 & 0 & 0.0 & 0.392 \\
\hline Anomia & 1 & 0.9 & 1 & 1.6 & 0 & 0.0 & 0.392 \\
\hline
\end{tabular}

$N$ number, \% percentage

*Used tests chi-square and Fisher exact tests

The higher mean score for repetition at the onset was for aphasia without repetitive disorder more than aphasia with repetitive disorder, and better improvement after 1 and 3 months (regarding repetition, comprehension, spontaneous speech, naming, reading, and writing) was for aphasia without repetitive disorder (Table 3 and Fig. 1).

The scoring of KAAT test at the onset and follow-up study between the most three frequent types of aphasia among the patients (global, Broca's, and subcortical aphasia) showed that the highest mean score at the onset was for Broca's aphasia. And the best improvement after 1 and 3 months (regarding repetition, comprehension, spontaneous speech, naming, reading, and writing) was for subcortical aphasia then Broca's aphasia, and the least improvement was for global aphasia (Table 4 and Fig. 2).

On comparing demographic and neuroimaging data regarding mean score of KAAT at the onset and rate of recovery of aphasia in the follow-up study, no statistically significant difference was reported between patients regarding different age groups or sex or size or pathology of lesion at the onset. But in the follow-up study, better improvement

Table 2 Neuroimaging

\begin{tabular}{lll}
\hline Neuroimaging & $N(107)$ & $\%$ \\
\hline Cerebral hemorrhage & 21 & 19.6 \\
Cerebral infarction & 86 & 80.4 \\
Size of infarction: & & \\
1- Small & 9 & 8.4 \\
2- Medium & 6 & 5.6 \\
3- Large & 71 & 66.4 \\
\hline
\end{tabular}

$N$ number, \% percentage was reported among younger age groups, patients with cerebral hemorrhage, patients with subcortical aphasia, and patients with small-sized lesions (Table 5).

\section{Discussion}

There is no sufficient data about aphasia epidemiology in Egypt, and few studies was conducted about that but in this study relative frequency of PSA in Assiut University Hospital was 7.1\%, and this nearly agreed with El-Tallawy and colleagues, 2015, a previous study done at Assiut, which reported PSA among 6.7\% of stroke patients [14].

In present study, aphasia was more common among male patients $(57.9 \%)$ than among female patients (42.1\%) and this was consistent with Gerges [15]. On the other hand, Engelter and colleagues [9], Kyrozis and

Table 3 Comparing KAAT score at onset between aphasia with repetitive disorder and without repetitive disorder

\begin{tabular}{llll}
\hline KAAT score & \multicolumn{2}{l}{ Types of aphasia } & $P$ value \\
\cline { 2 - 3 } & $\begin{array}{l}\text { With repetitive } \\
\text { disorder } \\
\text { Mean } \pm \text { SD }\end{array}$ & $\begin{array}{l}\text { Without repetitive } \\
\text { disorder } \\
\text { Mean } \pm \text { SD }\end{array}$ & \\
\hline Repetition & $0.28 \pm 0.81$ & $2.00 \pm 3.69$ & $0.040^{*}$ \\
Comprehension & $2.04 \pm 4.64$ & $1.23 \pm 3.53$ & 0.503 \\
Spontaneous speech & $5.69 \pm 6.21$ & $4.77 \pm 9.22$ & 0.579 \\
Naming & $0.08 \pm 0.28$ & $0.00 \pm 0.00$ & 0.166 \\
Reading & $0.00 \pm 0.00$ & $0.18 \pm 0.66$ & 0.061 \\
Writing & $0.09 \pm 0.77$ & $0.45 \pm 1.74$ & 0.133 \\
Total score & $8.20 \pm 9.49$ & $8.23 \pm 17.15$ & 0.992 \\
\hline KAAT Kasr El-Eini Arabic Aphasia test, SD standard deviation & \\
*Significant $P$ value at $<0.05$, used test Mann-Whitney test &
\end{tabular}




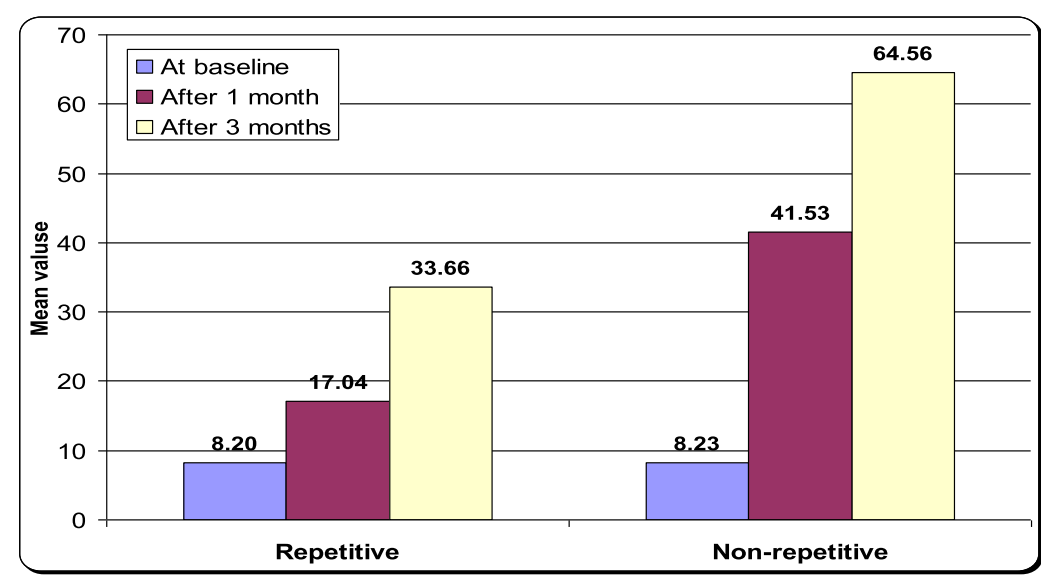

P-value: at onset 0.992

after1 month $0.000 * *$

after 3 months $0.000 * *$

** Highly significant P value at $<0.01$, used tests Wilcoxon Signed Rank Test, Mann-Whitney test, KAAT: Kasr El-Eini Arabic Aphasia test

Fig. 1 Comparing KAAT score at follow-up between aphasia with repetitive disorder and without repetitive disorder

colleagues [16], and Sinanović and colleagues [17] reported that aphasia was more frequent among females. However Kang and colleagues reported no sex difference in the incidence of aphasia [18]. In the present study, aphasia was more among males which can be explained that stroke patients who were admitted to Assiut University Hospital during the time of study were more males.

In present study, there was no significant difference between males and females regarding clinical types of aphasia. This was consistent with other studies that reported there was no association between gender and aphasia type specifically, Godefroy and colleagues [19] and Engelter and colleagues [9] who observed no gender differences on measures of auditory comprehension and expressive language.
In present study, there was no significant difference between males and females regarding initial aphasia score or rate of recovery and that could be explained that both genders have the same mechanisms for aphasia recovery as regards the restoration of brain perfusion or neuroplasticity changes in the brain [20]. This was consistent with studies by Inatomi and colleagues [21], Seniow and colleagues [22], and Watila and colleague [23] that reported no gender difference in aphasia recovery.

The studied patients were classified into two groups, aphasia with repetitive disorder (79.4\%) "Including global, Broca's, Wernicke, conduction aphasia and aphemia" and aphasia without repetitive disorder (20.6\%) "Including striatal, thalamic, mixed transcortical, transcortical motor, transcortical sensory and anomia".

Table 4 Comparing KAAT score at onset between the most frequent aphasia subtypes (global, Broca's, and subcortical)

\begin{tabular}{|c|c|c|c|c|c|c|}
\hline \multirow[t]{2}{*}{ KAAT score } & \multicolumn{3}{|c|}{ Types of aphasia } & \multirow[t]{2}{*}{$P$ value ${ }^{1}$} & \multirow[t]{2}{*}{$P$ value ${ }^{2}$} & \multirow[t]{2}{*}{$P$ value } \\
\hline & Global & $\begin{array}{l}\text { Broca's } \\
\text { Mean } \pm \text { SD }\end{array}$ & Subcortical & & & \\
\hline Repetition & $0.06 \pm 0.33$ & $1.44 \pm 1.42$ & $0.06 \pm 0.24$ & $0.000^{* *}$ & 0.556 & $0.003^{* *}$ \\
\hline Comprehension & $0.23 \pm 0.80$ & $13.78 \pm 1.64$ & $0.00 \pm 0.00$ & $0.000^{* *}$ & 0.217 & $0.000^{* *}$ \\
\hline Spontaneous speech & $4.20 \pm 3.31$ & $7.67 \pm 1.22$ & $1.06 \pm 2.54$ & $0.002^{* *}$ & $0.000^{* *}$ & $0.000^{* *}$ \\
\hline Naming & $0.03 \pm 0.17$ & $0.33 \pm 0.50$ & $0.00 \pm 0.00$ & $0.000^{* *}$ & 0.486 & $0.013^{*}$ \\
\hline Reading & $0.00 \pm 0.00$ & $0.00 \pm 0.00$ & $0.00 \pm 0.00$ & 1.000 & 1.000 & 1.000 \\
\hline Writing & $0.00 \pm 0.00$ & $0.11 \pm 0.33$ & $0.00 \pm 0.00$ & $0.005^{* *}$ & 1.000 & 0.169 \\
\hline Total score & $4.52 \pm 3.78$ & $23.33 \pm 3.46$ & $0.59 \pm 1.80$ & $0.000^{* *}$ & $0.000^{* *}$ & $0.000^{* *}$ \\
\hline
\end{tabular}

KAAT Kasr El-Eini Arabic Aphasia test, SD standard deviation

${ }^{1}$ Comparison between global and Broca's

${ }^{2}$ Comparison between global and subcortical

${ }^{3}$ Comparison between Broca's and subcortical aphasia

*Significant $P$ value at $<0.05,{ }^{* *}$ highly significant at $<0.01$, used test Kruskal Wallis Test 


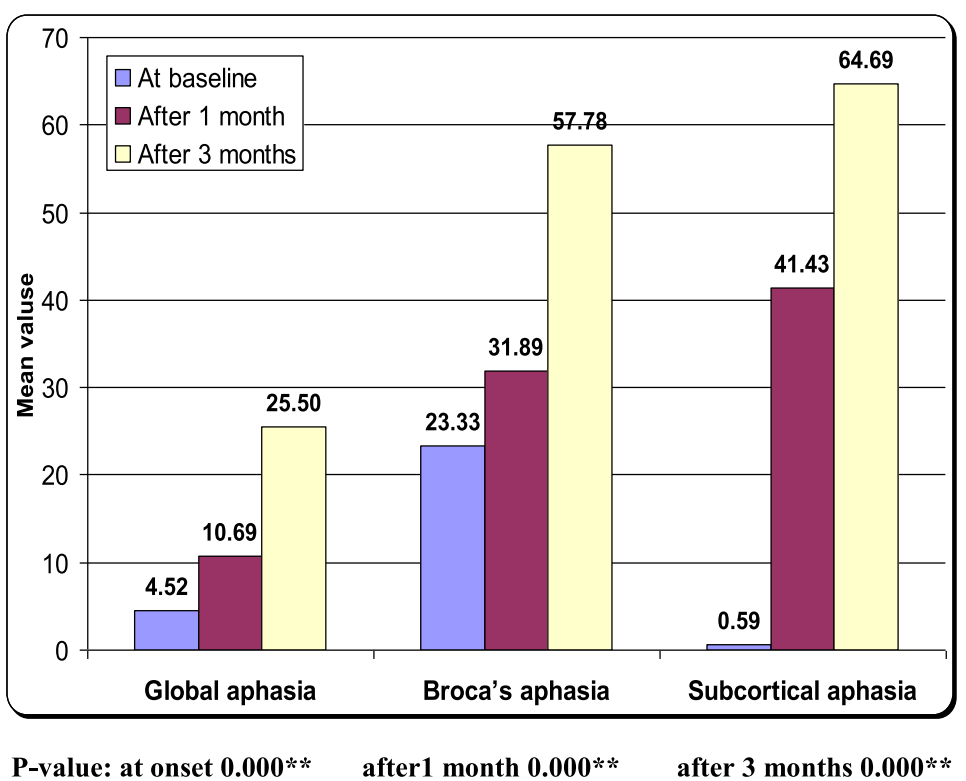

** Highly significant $\mathrm{P}$ value at $<0.01$, used tests Wilcoxon Signed Rank Test and Kruskal Wallis Test, KAAT: Kasr El-Eini Arabic Aphasia test

Fig. 2 Comparing KAAT score at follow-up for the most frequent aphasia subtypes (global, Broca's, and subcortical)

The most frequent type of aphasia was global aphasia $(66.4 \%)$, followed by striatal (11.2\%), Broca's $(8.4 \%)$, thalamic (4.7\%), Wernicke (2.8\%), mixed transcortical (1.9\%), conduction, transcortical motor, transcortical sensory, anomia, and aphemia $(0.9 \%$ for each type of them). Many other studies had variable results regarding aphasia subtypes which could be attributed to variations in methodology.

The most frequent type of aphasia was global aphasia (66.4\%) which could be explained that this study was carried out among the inpatients of Assiut University Hospital which is considered as a main center at Upper Egypt where most critical cases (as patients with global aphasia) are admitted because there is lack of neurology departments in Upper Egypt.

Higher score for repetition at the onset was reported for aphasia without repetitive disorder than aphasia with repetitive disorder, and there was better recovery during the follow-up in aphasia without repetitive disorder regarding repetition, comprehension, spontaneous speech, naming, reading, and writing. Then, comparative assessment was done between the most frequent three types of aphasia in the studied patients including global (66.4\%), subcortical "Thalamic and striatal aphasia" (15.9\%), and Broca's aphasia (8.4\%).

These results revealed that the highest initial mean score at the onset was for Broca's aphasia while the best recovery during the follow-up (regarding repetition, comprehension, spontaneous speech, naming, reading, and writing) was for subcortical aphasia $(p=0.000)$ then Broca's aphasia, with the least recovery for global aphasia.

This was consistent with Pedersen and colleagues, and Jung and colleagues who observed that those with global aphasia had poorer recovery than those with other aphasia types, which may reflect higher stroke severity and extensive lesions that affect brain areas responsible for language process [24, 25].Also Kang and colleagues reported better recovery in patients with subcortical aphasia which can be explained that patients with subcortical aphasia have preserved cortical structures with their ability for neuroplasticity changes that can help for aphasia recovery [18].

In comparing demographic, clinical, and neuroimaging data regarding the rate of recovery of aphasia in the follow-up study, it was reported that there were many prognostic factors that can predict rate of aphasia recovery.

There was no significant difference between different age groups regarding initial aphasia score at the onset. This was consistent with Kang and colleagues who found no difference between age and initial aphasia severity [18], but better recovery during follow-up was reported among younger age groups $(p=0.041)$ which can be explained that older patients had structural changes through aging process that impair mechanisms of aphasia recovery. This was consistent with Laska and colleagues and Watila and colleague who reported that there is a tendency for older patients to have a poorer recovery [23, 26], 
Table 5 Comparing demographic, clinical, and neuroimaging data regarding rate of aphasia recovery

\begin{tabular}{|c|c|c|c|c|}
\hline \multicolumn{5}{|c|}{ Percentage of improvement of total KAAT score } \\
\hline & \multicolumn{3}{|l|}{ Age groups } & \multirow[t]{3}{*}{$P$ value $^{1}$} \\
\hline & $20-<40$ & $40-<60$ & $\geq 60$ & \\
\hline & \multicolumn{3}{|l|}{ Mean \pm SD } & \\
\hline At onset & $13.84 \pm 13.14$ & $14.16 \pm 19.92$ & $10.16 \pm 13.23$ & 0.369 \\
\hline After (1)month & $25.88 \pm 28.59$ & $15.71 \pm 9.36$ & $13.78 \pm 18.37$ & 0.116 \\
\hline \multirow[t]{4}{*}{ After (3) months } & $50.75 \pm 31.60$ & $40.88 \pm 16.57$ & $25.55 \pm 15.73$ & $0.041^{*}$ \\
\hline & \multicolumn{2}{|c|}{ Pathology of stroke } & & $P$ value $^{2}$ \\
\hline & hemorrhagic & Ischemic & & \\
\hline & Mean \pm SD & Mean \pm SD & & \\
\hline At onset & $8.95 \pm 7.34$ & $10.94 \pm 11.73$ & & 0.467 \\
\hline After (1)month & $38.34 \pm 30.56$ & $11.35 \pm 12.95$ & & $0.010^{* *}$ \\
\hline \multirow[t]{4}{*}{ After (3) months } & $64.08 \pm 36.83$ & $35.19 \pm 20.27$ & & $0.019^{*}$ \\
\hline & \multicolumn{2}{|c|}{ Size of infarction } & & $P$ value ${ }^{1}$ \\
\hline & Small & Medium & Large & \\
\hline & \multicolumn{3}{|l|}{ Mean \pm SD } & \\
\hline At onset & $12.32 \pm 14.54$ & $10.32 \pm 9.32$ & $8.44 \pm 8.18$ & 0.446 \\
\hline After (1)month & $23.81 \pm 26.59$ & $11.84 \pm 10.51$ & $9.08 \pm 7.82$ & 0.338 \\
\hline \multirow[t]{4}{*}{ After (3) months } & $52.00 \pm 29.63$ & $36.79 \pm 17.19$ & $32.02 \pm 17.43$ & $0.031^{*}$ \\
\hline & \multicolumn{2}{|l|}{ Site of lesion } & & $P$ value ${ }^{2}$ \\
\hline & Cortical & Subcortical & & \\
\hline & Mean \pm SD & Mean \pm SD & & \\
\hline At onset & $5.53 \pm 4.36$ & $0.59 \pm 1.80$ & & $0.000^{* *}$ \\
\hline After (1)month & $8.59 \pm 7.61$ & $41.43 \pm 15.08$ & & $0.000^{* *}$ \\
\hline \multirow[t]{4}{*}{ After (3) months } & $31.48 \pm 16.83$ & $64.69 \pm 14.67$ & & $0.000^{* *}$ \\
\hline & \multicolumn{2}{|l|}{ Sex } & & $P$ value ${ }^{2}$ \\
\hline & Males & Females & & \\
\hline & Mean \pm SD & Mean \pm SD & & \\
\hline At onset & $11.03 \pm 14.99$ & $11.37 \pm 14.68$ & & 0.975 \\
\hline After (1)month & $16.24 \pm 21.07$ & $19.66 \pm 22.36$ & & 0.170 \\
\hline After (3) months & $38.39 \pm 28.15$ & $44.74 \pm 25.26$ & & 0.062 \\
\hline
\end{tabular}

KAAT Kasr El-Eini Arabic Aphasia test, SD standard deviation

${ }^{1}$ Used Kruskal Wallis test

${ }^{2}$ Used Mann-Whitney test

* Significant $P$ value at $<0.05$

**Highly significant at $<0.01$

while other studies such as Inatomi and colleagues [21] reported age as a not significant prognostic indicator for aphasia recovery which could be explained by short follow-up period in their study.

Better recovery was reported with hemorrhagic stroke more than ischemic stroke ( $p=0.010$ after one month and $p=0.019$ after three months). This was consistent with Jung and colleagues who found that hemorrhagic stroke survivors had a better prognosis than ischemic stroke patients. The better prognosis may be due to fiber bundles being displaced without damage in hemorrhagic strokes [25].
In the present study, there was a negative correlation between the size of cerebral infarction and recovery rate as better recovery was for small-sized infarction $(p=0.031)$. This was consistent with Maas and colleagues [27] and Henseler and colleagues [28] who reported the negative influence of larger lesion on PSA recovery.

The limitation of this study is that a larger sample size with longer duration follow-up can be included with further assessment with functional neuroimaging to study the role of cerebral hemispheres in language recovery. 


\section{Conclusion}

Relative frequency of PSA in Assiut University Hospital during the 6 -month period was $7.1 \%$ with the most frequent type of aphasia being global subtype, and there were many prognostic factors which can predict rate of recovery in PSA like age of the patient, aphasia type, size, site, and pathology of the lesion. So, further assessment of PSA with a larger sample size and longer duration are recommended.

\section{Abbreviations}

CT: Computed tomography; KAAT: Kasr El-Eini Arabic Aphasia test; MRI: Magnetic resonance imaging, $N$ : Number; PSA: Post-stroke aphasia; SD: Standard deviation

\section{Acknowledgements}

Not applicable.

\section{Funding}

Not applicable.

\section{Availability of data and materials}

The paper is a part of a thesis conducted in Faculty of Medicine, Assiut University, and approved by its ethical committee. The data can be publicly available at the Faculty of Medicine, Assiut University.

\section{Authors' contributions}

$\mathrm{HN}$ and $\mathrm{AH}$ participated in the design of the study, guidance, follow-up, and final revision. AM helped in the practical works, participated in the design of the study, and participated in the statistical analysis. MN contributed to the acquisition, analysis, and interpretation of the data; performed the statistica analysis; prepared the paper; participated in the sequence alignment; and drafted the final revision of the manuscript. All authors read and approved the final manuscript.

\section{Ethics approval and consent to participate}

An informed consent was obtained from all the patients before participating in the study. Assiut Faculty of Medicine Ethical committee approved this study in May 24, 2015. The confidentiality of the patients' information was maintained during all the steps of the study.

\section{Consent for publication}

Not applicable.

\section{Competing interests}

The authors declare that they have no competing interests.

\section{Publisher's Note}

Springer Nature remains neutral with regard to jurisdictional claims in published maps and institutional affiliations.

\section{Author details}

${ }^{1}$ Neurology Department, Faculty of Medicine, Assiut University, Assiut, Egypt.

${ }^{2}$ Neurology Department, Faculty of Medicine, Cairo University, Giza, Egypt.

Received: 28 December 2018 Accepted: 22 May 2019

Published online: 07 June 2019

\section{References}

1. National Aphasia Association. Aphasia definitions. Available from: https:// www.aphasia.org/aphasia-definitions/2017. Accessed 24 Jan 2018.

2. McNeil M, Pratt S. Defining aphasia: some theoretical and clinical implications of operating from a formal definition. Aphasiology. 2001:15(10/ 11):901-11.

3. Hilari K. The impact of stroke: are people with aphasia different to those without? Disabil Rehabil. 2011;33(3):211-8
4. El Hachioui H, Lingsma HF, van de Sandt-Koenderman ME, Dippel DW, Koudstaal PJ, Visch-Brink EG. Recovery of aphasia after stroke: a 1-year follow-up study. J Neurol. 2013;260(1):166-71.

5. Davidson B, Howe T, Worrall L, Hickson L, Togher L. Social participation for older people with aphasia: the impact of communication disability on friendships. Top Stroke Rehabil. 2008;15(4):325-40.

6. Dickey L, Kagan A, Lindsay MP, Fang J, Rowland A, Black S. Incidence and profile of inpatient stroke-induced aphasia in Ontario, Canada. Arch Phys Med Rehabil. 2010;91(2):196-202.

7. Feigin VL, Forouzanfar MH, Krishnamurthi R, Mensah GA, Connor M, Bennett DA, et al. Global and regional burden of stroke during 1990-2010: findings from the Global Burden of Disease Study 2010. Lancet. 2014;383(9913):245-54.

8. Ellis C, Urban S. Age and aphasia: a review of presence, type, recovery and clinical outcomes. Top Stroke Rehabil. 2016;23(6):430-9.

9. Engelter ST, Gostynski M, Papa S, Frei M, Born C, Ajdacic-Gross V, et al. Epidemiology of aphasia attributable to first ischemic stroke: incidence, severity, fluency, etiology, and thrombolysis. Stroke. 2006;37(6):1379-84.

10. Alexander MP, Benson DF. The aphasias and related disturbance. In: Joynt R, editor. Clinical Neurology, vol. 1. Philadelphia: J.B, Lippincott; 1997.

11. Hassanein A, El-Tamawy M, Sallam T, Hosny H, Abdel Naseer M, El-Fayoumy N. Kasr El-Aini Arabic Aphasia test (KAAT) simple, standarized, valid, reliable test for Egyptian patients, literate and illiterate. Egypt J Neurol Psychiatry Neurosurg. 2002;39:381-95.

12. Benson DF, Geschwind N. The aphasias and related disturbances. In: Backer AB, Backer LH, editors. Clinical Neurology,Vol1,chapter 8. New York: Harper and Row; 1984.

13. Brott T, Marler JR, Olinger CP, Adams HP, Tomsick T, Barsan WG, et al. Measurements of acute cerebral infarction: lesion size by computed tomography. Stroke. 1989;20(7):871-5.

14. El Tallawy HN, Farghaly WM, Badry R, Hamdy NA, Shehata GA, Rageh TA, et al. Epidemiology and clinical presentation of stroke in Upper Egypt (desert area). Neuropsychiatr Dis Treat. 2015;11:2177-83.

15. Gerges M. Diagnostic and prognostic correlates of Extracranial and Intracranial Duplex Ultrasonography in Vascular Aphasia. M.D. Thesis in Neurology, Faculty of Medicine, Cairo University. 2011/ Available from http://erepository.cu.edu.eg/index.php/cutheses/thesis/view/7279. Accessed 24 Jan 2018.

16. Kyrozis A, Potagas C, Ghika A, Tsimpouris PK, Virvidaki ES, Vemmos KN. Incidence and predictors of post-stroke aphasia: the Arcadia Stroke Registry. Eur J Neurol. 2009;16(6):733-9.

17. Sinanović O, Mrkonjić Z, Zukić S, Vidović M, Imamović K. Poststroke language disorders. Acta Clin Croat. 2011;50:79-94.

18. Kang EK, Sohn HM, Han MK, Kim W, Han TR, Paik NJ. Severity of post-stroke aphasia according to aphasia type and lesion location in Koreans. J Korean Med Sci. 2010;25(1):123-7.

19. Godefroy O, Dubois C, Debachy B, Leclerc M, Kreisler A, Lille Stroke P. Vascular aphasias: main characteristics of patients hospitalized in acute stroke units. Stroke. 2002:33(3):702-5.

20. Kiran S. What is the nature of poststroke language recovery and reorganization? ISRN Neurol. 2012;2012:786872.

21. Inatomi $Y$, Yonehara T, Omiya S, Hashimoto Y, Hirano T, Uchino M. Aphasia during the acute phase in ischemic stroke. Cerebrovasc Dis. 2008;25(4):316-23.

22. Seniow J, Litwin M, Lesniak $M$. The relationship between non-linguistic cognitive deficits and language recovery in patients with aphasia. J Neurol Sci. 2009:283(1-2):91-4.

23. MM W, SA B. Factors predicting post-stroke aphasia recovery. J Neurol Sci. 2015;352:12-8.

24. Pedersen PM, Vinter K, Olsen TS. Aphasia after stroke: type, severity and prognosis. The Copenhagen aphasia study. Cerebrovasc Dis. 2004:17(1):35-43.

25. Jung IY, Lim JY, Kang EK, Sohn HM, Paik NJ. The factors associated with good responses to speech therapy combined with transcranial direct current stimulation in post-stroke aphasic patients. Ann Rehabil Med. 2011; 35(4):460-9.

26. Laska AC, Hellblom A, Murray V, Kahan T, Von Arbin M. Aphasia in acute stroke and relation to outcome. J Intern Med. 2001;249(5):413-22.

27. Maas MB, Lev MH, Ay H, Singhal AB, Greer DM, Smith WS, et al. The prognosis for aphasia in stroke. J Stroke Cerebrovasc Dis. 2012;21(5):350-7.

28. Henseler I, Regenbrecht F, Obrig H. Lesion correlates of patholinguistic profiles in chronic aphasia: comparisons of syndrome-, modality- and symptom-level assessment. Brain. 2014;137(Pt 3:918-30. 